\title{
Monocyte subsets and their activation markers in Kawasaki disease - A Study from a Tertiary care Center in North India
}

\section{Aarchie Gupta}

Post Graduate Institute of Medical Education and Research

\section{Kaki Sairam}

Post Graduate Institute of Medical Education and Research

Pandiarajan Vignesh ( $\square$ vigimmc@gmail.com )

Post Graduate Institute of Medical Education and Research

\section{Archan Sil}

Post Graduate Institute of Medical Education and Research

\section{Jhumki Das}

Post Graduate Institute of Medical Education and Research

\section{Kanika Arora}

Post Graduate Institute of Medical Education and Research

Jitendra K. Shandilya

Post Graduate Institute of Medical Education and Research

\section{Rajni Kumrah}

Post Graduate Institute of Medical Education and Research

\section{Deepti Suri}

Post Graduate Institute of Medical Education and Research

\section{Amit Rawat}

Post Graduate Institute of Medical Education and Research

\section{Surjit Singh}

Post Graduate Institute of Medical Education and Research

\section{Research Article}

Keywords: Monocyte activation, Children, Kawasaki disease

Posted Date: February 16th, 2022

DOI: https://doi.org/10.21203/rs.3.rs-1307416/v1 
License: (c) (i) This work is licensed under a Creative Commons Attribution 4.0 International License. Read Full License 


\section{Abstract}

Background: Kawasaki disease (KD) is a common childhood vasculitis and autopsy studies have shown monocyte infiltration in affected blood vessels. Role of monocyte subsets and their activation status in KD have not been completely explored.

Materials and methods: Sixteen children diagnosed with KD were included in our study along with 16 age and sex-matched controls. We studied subpopulations of monocytes (classical, intermediate and nonclassical), early and late monocyte activation markers during acute and convalescent stages. We also assessed soluble markers of monocyte activation (sCD14, SCD163, CCL2).

Results: Significant elevation in absolute number of classical [CD14+CD16-] and intermediate monocytes [CD14++CD16+] were noted in KD and febrile controls in comparison to healthy controls. CD69 expression in classical, intermediate and non-classical monocytes were higher in KD when compared to healthy controls. Absolute counts of classical and intermediate monocytes expressing CD69 and HLA-DR were significantly lower in the convalescent stage as compared to acute KD. We observed no significant elevation in SCD14 and SCD163 levels in KD as compared to controls. However, median CCL2 levels were highest in children with KD.

Conclusion: Our results suggest that both early and late monocyte activation occur in acute phase of KD that subsides in convalescent phase.

\section{Introduction}

Kawasaki disease (KD) is a common childhood medium vessel vasculitis predominantly affecting coronary arteries. [1] KD is now considered the most common cause of acquired heart disease in children in developed countries. Around $20-25 \%$ of untreated children develop coronary artery abnormalities (CAAs) in KD. The disease has gained more attention in recent times due to the overlapping features with multisystem inflammatory syndrome in children (MIS-C) temporally associated with severe acute respiratory syndrome corona virus-2 (SARS-CoV-2). [2] Children of Asian ethnicity are more commonly affected than Africans, Hispanics, and Caucasians. Etiology of KD is currently unknown. KD is likely triggered by an infectious or environmental agent that initiates an abnormal immune response in a genetically susceptible host. [3]

Activated monocytes play an important role in the immunopathogenesis of KD by production of proinflammatory cytokines such as TNF-a (tumor necrosis factor), IL1 $\beta$ (interleukin), and IL-6. In the acute stage of KD, increase in circulating CD14+ monocyte counts have been described. Recent studies have documented that CD14+CD16++ monocytes (non-classical monocyte subpopulation) play an important role in inflammation in KD [4]. CD14 along with toll-like receptor 4 (TLR4) functions as a coreceptor for binding to the bacterial lipopolysaccharides (LPS). Several studies have also noted increased levels of circulating soluble monocyte activation markers like SCD14, sCD163, MCP-1 and CCL-2 in acute stages of KD. [5-8] Although individual markers of monocytes activation have been studied in children with KD, 
combination of different markers of monocyte activation has not been assessed yet. There is also paucity of literature on different monocyte subpopulations in KD.

In this study, we studied peripheral blood levels of monocyte activation markers (including early and late markers) and different populations of monocytes (classical [CD14+CD16-], intermediate [CD14++CD16+], and non-classical [CD14+CD16++] monocytes) in acute KD, and also in follow-up to assess the impact of intravenous immunoglobulin (IVIg) therapy on monocyte activation.

\section{Results}

Sixteen $(n=16)$ children diagnosed with KD were included in the present study. Sixteen children were taken as febrile controls and 16 as healthy controls. The median age group [interquartile range (IQR)] of children with KD was 1.95 years (0.6-4.25) as compared to 2 (1.25-5.5) and 3 (1.25-5.5) years in febrile controls and healthy controls respectively. Male to female ratio of cases, febrile controls, and healthy controls was 2.2:1, 1.6:1, and 0.7:1 respectively. All patients with KD had a fever duration of more than 5 days. Median fever duration in children with KD and febrile controls were 9 (7-15) and 7.5 (6.5-18) days, respectively (Table 1). Causes of fever in febrile controls included pneumonia in 3 , liver abscess in 3 , tuberculosis in 3, empyema in 1, enteric fever in 1, urinary tract infection in 1, meningitis in 1, cellulitis in 1 , osteomyelitis in 1, and dengue in 1. Among cases with KD, 7 (43\%) had incomplete KD and $9(56 \%)$ had complete KD. None had features of shock or multisystem inflammatory syndrome temporally associated with severe acute respiratory syndrome corona virus-2 (SARS CoV-2) infection. Three patients had evidence of CAAs - 2 had persistent and 1 had transient CAA (Suppl. Table). All children with KD received IVlg $2 \mathrm{~g} / \mathrm{kg}$, 3 children received Infliximab, 2 received cyclosporin, 1 received glucocorticoids.

Table 1

Baseline clinical characteristics of children with Kawasaki disease at enrolement $(n=16)$

\begin{tabular}{|ll|}
\hline Clinical Parameters & Results \\
\hline Median age (IQR) & 1.95 years (0.6-4.25) \\
\hline Male: Female & $2.2: 1$ \\
\hline No of cases of incomplete KD & $7(43 \%)$ \\
\hline Fever $\geq 5$ days & $16(100.0 \%)$ \\
\hline Changes in extremities & $14(87.5 \%)$ \\
\hline Polymorphus exanthem & $9(56.2 \%)$ \\
\hline Conjunctival injection & $16(100.0 \%)$ \\
\hline Changes in lips and oral cavity & $12(75.0 \%)$ \\
\hline Cervical lymphadenopathy $(>1.5 \mathrm{~cm})$ & $11(68.8 \%)$ \\
\hline
\end{tabular}


Comparison of monocyte subpopulations and monocyte activation markers (by Flow cytometry)

Eleven ( $n=11$ ) out of $16 \mathrm{KD}$ children underwent flow-cytometry for assessment of monocyte subpopulations and expression of monocyte activation markers (CD69 and HLA-DR). Children with acute KD and febrile controls had a significant elevation in absolute monocyte count (AMC) compared to healthy controls. Significant elevation in absolute number of classical [CD14+CD16-] and intermediate monocytes [CD14++CD16+] were also noted in KD and febrile controls in comparison to healthy controls (Table 2) (Figure 2). 
Table 2

Comparison of leukocyte, monocyte counts, monocyte subsets and monocyte activation markers between children with $\mathrm{KD}$, febrile, and healthy controls.

\begin{tabular}{|c|c|c|c|c|c|}
\hline Parameters & $\begin{array}{l}\text { Acute KD } \\
(n=11) \\
\text { [Median } \\
\text { (IQR)] }\end{array}$ & $\begin{array}{l}\text { Febrile Control } \\
(n=11) \\
\text { [Median (IQR)] }\end{array}$ & $\begin{array}{l}\text { Healthy } \\
\text { Control (n= } \\
\text { 11) [Median } \\
\text { (IQR)] }\end{array}$ & \multicolumn{2}{|l|}{ p- value* } \\
\hline \multirow{3}{*}{$\begin{array}{l}\text { TLC (Total Leukocyte } \\
\text { Count) }\end{array}$} & 14440.00 & 15000.00 & 9670.00 & 0.018 & \\
\hline & (10105.00- & (11485.00- & (8720.00- & & \\
\hline & 26100.00) & 18710.00) & 10145.00) & & \\
\hline AMC (Absolute & $\begin{array}{l}1554.90 \\
(774.60-\end{array}$ & 1320 (691.04- & $\begin{array}{l}518.52 \\
(448.55-\end{array}$ & 0.007 & \\
\hline monocyte count) & 1998.32) & 1389.92) & 599.13) & & \\
\hline ALC (Absolute & 6041.60 & 4387.60 & 3321.00 & 0.045 & \\
\hline \multirow[t]{2}{*}{ lymphocyte count) } & $(4118.60-$ & $\begin{array}{l}(3025.50- \\
5923)\end{array}$ & (2931.90- & & \\
\hline & 7921.80) & & $3890.00)$ & & \\
\hline Classical monocytes & $\begin{array}{l}1288.77 \\
(443.61-\end{array}$ & $\begin{array}{l}588.16 \\
(412.39-\end{array}$ & $\begin{array}{l}367.72 \\
(254.85-\end{array}$ & 0.029 & \\
\hline [CD14+CD16-] & 1435.51) & $763.28)$ & 404.19) & & \\
\hline \multicolumn{6}{|l|}{ (absolute counts) } \\
\hline Classical monocytes & 70.87 (5.57- & & $49.56(45.29-$ & $\begin{array}{l}71.59 \\
(53.61-\end{array}$ & 0.368 \\
\hline [CD14+CD16-] (\%) & 77.71) & & 65.68) & 73.96) & \\
\hline Intermediate monocytes & $\begin{array}{l}114.75 \\
(59.77-\end{array}$ & 97.31 (35.26- & 13.96 (10.47- & 0.007 & \\
\hline [CD14++CD16] & 158.11) & 164.79) & 37.96) & & \\
\hline \multicolumn{6}{|l|}{ (absolute counts) } \\
\hline Intermediate monocytes & $6.02(4.81-8.87)$ & & $8.45(4.42-$ & $\begin{array}{l}2.91 \\
(1.85- \\
6.52)\end{array}$ & 0.086 \\
\hline [CD14++CD16+] (\%) & & & 17.13) & & \\
\hline Non-classical monocytes & 48.41 (27.69- & 60.12 (48.08- & $40.44(26.22-$ & 0.676 & \\
\hline [CD14+CD16++] & 100.47) & 101.71) & 129.67) & & \\
\hline
\end{tabular}




\begin{tabular}{|c|c|c|c|c|c|}
\hline Parameters & $\begin{array}{l}\text { Acute KD } \\
(n=11) \\
\text { [Median } \\
\text { (IQR)] }\end{array}$ & $\begin{array}{l}\text { Febrile Control } \\
(n=11) \\
\text { [Median (IQR)] }\end{array}$ & $\begin{array}{l}\text { Healthy } \\
\text { Control ( } n= \\
\text { 11) [Median } \\
\text { (IQR)] }\end{array}$ & \multicolumn{2}{|c|}{ p-value* } \\
\hline Non-classical monocytes & $6.65(3.09-8.25)$ & & $\begin{array}{l}7.08(3.88- \\
9.37)\end{array}$ & $\begin{array}{l}9.64 \\
(5.84-\end{array}$ & 0.434 \\
\hline [CD14+CD16++] (\%) & & & & 18.43) & \\
\hline $\begin{array}{l}\text { CD69 expression in CD14+ } \\
\text { monocytes } \\
\text { (absolute counts) }\end{array}$ & $\begin{array}{l}191.82 \\
(83.01- \\
314.12)\end{array}$ & $\begin{array}{l}142.81(107.12- \\
175.33)\end{array}$ & $\begin{array}{l}35.90(27.98- \\
52.99)\end{array}$ & 0.001 & \\
\hline \multicolumn{2}{|c|}{ CD69 expression in CD14+ monocytes (\%) } & $\begin{array}{l}11.67 \text { (9.47- } \\
15.84)\end{array}$ & $\begin{array}{l}11.94(9.72- \\
15.61)\end{array}$ & $\begin{array}{l}7.36 \\
(5.28- \\
8.63)\end{array}$ & 0.031 \\
\hline $\begin{array}{l}\text { CD69 expression in } \\
\text { Classical } \\
\text { Monocytes [CD14+CD16-] } \\
\text { (absolute counts) }\end{array}$ & $\begin{array}{l}99.94(7.24- \\
213.30)\end{array}$ & $\begin{array}{l}37.40(21.17- \\
74.30)\end{array}$ & $\begin{array}{l}11.55(9.00- \\
13.18)\end{array}$ & 0.006 & \\
\hline $\begin{array}{l}\text { CD69 expression in } \\
\text { Classical } \\
\text { Monocytes [CD14+CD16-] } \\
\text { (\%) }\end{array}$ & $\begin{array}{l}10.44(7.24- \\
14.16)\end{array}$ & $\begin{array}{l}6.41(4.99- \\
10.94)\end{array}$ & $\begin{array}{l}3.83(2.37- \\
5.43)\end{array}$ & 0.009 & \\
\hline $\begin{array}{l}\text { CD69 expression in } \\
\text { Intermediate Monocytes } \\
\text { [CD14++CD16+] } \\
\text { (absolute counts) }\end{array}$ & $\begin{array}{l}9.19(4.55- \\
18.18)\end{array}$ & $\begin{array}{l}11.38(4.93- \\
20.18)\end{array}$ & $\begin{array}{l}1.78(0.74- \\
3.06)\end{array}$ & 0.004 & \\
\hline $\begin{array}{l}\text { CD69 expression in } \\
\text { Intermediate Monocytes } \\
\text { [CD14++CD16+] } \\
\text { (\%) }\end{array}$ & $\begin{array}{l}9.78(7.85- \\
14.83)\end{array}$ & $\begin{array}{l}15.29(9.51- \\
17.86)\end{array}$ & $\begin{array}{l}5.85(5.40- \\
13.55)\end{array}$ & 0.181 & \\
\hline $\begin{array}{l}\text { CD69 expression in Non- } \\
\text { classical Monocytes } \\
\text { [CD14+CD16++] } \\
\text { (absolute counts) }\end{array}$ & $\begin{array}{l}16.67(4.70- \\
24.93)\end{array}$ & $\begin{array}{l}19.97(6.85- \\
31.56)\end{array}$ & $\begin{array}{l}8.93(2.43- \\
17.17)\end{array}$ & 0.381 & \\
\hline $\begin{array}{l}\text { CD69 expression in Non- } \\
\text { classical Monocytes } \\
\text { [CD14+CD16++] } \\
\text { (\%) }\end{array}$ & $\begin{array}{l}24.75(18.10- \\
32.13)\end{array}$ & $\begin{array}{l}19.59(13.07- \\
40.62)\end{array}$ & $\begin{array}{l}10.20(7.66- \\
36.78)\end{array}$ & 0.445 & \\
\hline
\end{tabular}




\begin{tabular}{|c|c|c|c|c|c|}
\hline Parameters & $\begin{array}{l}\text { Acute KD } \\
\text { (n=11) } \\
\text { [Median } \\
\text { (IQR)] }\end{array}$ & $\begin{array}{l}\text { Febrile Control } \\
(n=11) \\
\text { [Median (IQR)] }\end{array}$ & $\begin{array}{l}\text { Healthy } \\
\text { Control }(n= \\
\text { 11) [Median } \\
\text { (IQR)] }\end{array}$ & p-value* & \\
\hline $\begin{array}{l}\text { HLA-DR expression in } \\
\text { CD14+ monocytes } \\
\text { (absolute counts) }\end{array}$ & $\begin{array}{l}783.83 \\
(441.86- \\
1020.86)\end{array}$ & $\begin{array}{l}537.97 \\
(347.30- \\
725.66)\end{array}$ & $\begin{array}{l}326.51 \\
(176.49- \\
346.48)\end{array}$ & 0.026 & \\
\hline HLA-DR expression in CD14+ & nonocytes (\%) & $\begin{array}{l}53.51(38.09- \\
68.41)\end{array}$ & $\begin{array}{l}45.62 \text { (35.36- } \\
70.61)\end{array}$ & $\begin{array}{l}54.84 \\
(47.56- \\
61.86)\end{array}$ & 0.974 \\
\hline $\begin{array}{l}\text { HLA-DR expression in } \\
\text { Classical } \\
\text { Monocytes [CD14+CD16-] } \\
\text { (absolute counts) }\end{array}$ & $\begin{array}{l}525.68 \\
(144.33- \\
660.41)\end{array}$ & $\begin{array}{l}241.83 \\
(146.44- \\
443.14)\end{array}$ & $\begin{array}{l}138.74 \\
(112.35- \\
202.21)\end{array}$ & 0.179 & \\
\hline $\begin{array}{l}\text { HLA-DR expression in } \\
\text { Classical } \\
\text { Monocytes [CD14+CD16-] } \\
(\%)\end{array}$ & $\begin{array}{l}50.16(12.83- \\
66.79)\end{array}$ & $\begin{array}{l}44.19(28.50- \\
92.59)\end{array}$ & $\begin{array}{l}52.59(34.23- \\
62.46)\end{array}$ & 0.899 & \\
\hline $\begin{array}{l}\text { HLA-DR expression in } \\
\text { Intermediate Monocytes } \\
\text { [CD14++CD16+] } \\
\text { (absolute counts) }\end{array}$ & $\begin{array}{l}97.91(48.01- \\
130.16)\end{array}$ & $\begin{array}{l}45.01(30.20- \\
125.08)\end{array}$ & $\begin{array}{l}9.72(5.01- \\
19.54)\end{array}$ & 0.017 & \\
\hline $\begin{array}{l}\text { HLA-DR expression in } \\
\text { Intermediate Monocytes } \\
\text { [CD14++CD16+] } \\
\text { (\%) }\end{array}$ & $\begin{array}{l}80.86(69.40- \\
88.44)\end{array}$ & $\begin{array}{l}75.58(42.23- \\
96.07)\end{array}$ & $\begin{array}{l}77.10(57.08- \\
86.33)\end{array}$ & 0.843 & \\
\hline $\begin{array}{l}\text { HLA-DR expression in Non- } \\
\text { classical Monocytes } \\
\text { [CD14+CD16++] } \\
\text { (absolute counts) }\end{array}$ & $\begin{array}{l}12.31(5.58- \\
37.19)\end{array}$ & $\begin{array}{l}18.70(11.93- \\
22.95)\end{array}$ & $\begin{array}{l}3.64(1.80- \\
8.45)\end{array}$ & 0.11 & \\
\hline $\begin{array}{l}\text { HLA-DR expression in Non- } \\
\text { classical Monocytes } \\
\text { [CD14+CD16++] } \\
\text { (\%) }\end{array}$ & $\begin{array}{l}35.64(26.53- \\
45.41)\end{array}$ & $\begin{array}{l}32.74(17.14- \\
44.69)\end{array}$ & $\begin{array}{l}11.76(4.16- \\
14.3)\end{array}$ & 0.12 & \\
\hline
\end{tabular}

Absolute counts of CD14+ monocytes expressing activation markers CD69 and HLA-DR were significantly high in KD and febrile controls as compared to healthy controls. However, CD69 expression in classical monocytes was significantly higher in acute KD, compared to febrile and healthy controls. CD69 expression in intermediate and non-classical monocytes were higher in KD when compared to healthy controls. However, the results are comparable with febrile controls. HLA-DR expression in classical and 
intermediate monocytes were higher in acute KD when compared to febrile and healthy controls. However, statistical significance was noted only with intermediate monocytes (Table 2) (Figure 2).

Flow cytometric parameters were also compared between acute KD, post-IVIg, and 3rd month follow-up samples in KD cases. Absolute counts of classical monocytes and intermediate monocytes were higher in acute KD and it progressively decreased in post-IVIg and 3rd month follow-up samples (Figure 3). The absolute counts of CD14+ monocytes expressing CD69 and absolute counts of CD14+ monocytes expressing HLA-DR were significantly higher in acute KD in comparison to 3rd month follow-up (Figure 3).

The absolute counts of classical [CD14+CD16-] and intermediate monocytes [CD14++CD16+] expressing CD69 and HLA-DR were significantly lower in the convalescent stage as compared to acute KD. CD69 expression in CD14+ monocytes was higher in KD patients with CAAs $(n=2)$ as compared to patients without CAAs ( $n=9)$. (Figure 3 )

\section{Comparison Of Monocyte Activation Markers (By Elisa)}

ELISA was done for 16 patients with KD to assess soluble activation markers of monocytes (SCD14, sCD163, CCL2). While median SCD14 and SCD163 levels were higher in febrile controls compared to children with KD and healthy controls, median CCL2 levels were highest in children with KD. (Table 3). We observed no significant fluctuations in levels of SCD14 and SCD163 over time in children with KD. However, levels of CCL2 significantly dropped after IVIg infusion in children with KD (Supplementary Table 1). 
Table 3

Comparison of monocyte and macrophage activation markers between children with KD, febrile, and healthy controls.

\begin{tabular}{|c|c|c|c|c|}
\hline & \multicolumn{3}{|l|}{ Group $(n=16)$} & \multirow{2}{*}{$\begin{array}{l}\text { p- } \\
\text { value* }\end{array}$} \\
\hline & $\begin{array}{l}\text { Children with KD (Acute phase) } \\
(n=16)\end{array}$ & $\begin{array}{l}\text { Febrile controls } \\
(n=16)\end{array}$ & $\begin{array}{l}\text { Healthy controls } \\
(n=16)\end{array}$ & \\
\hline & [Median (IQR)] & [Median (IQR)] & [Median (IQR)] & \\
\hline sCD14 & 2228.84 & 2754.10 & 2252.64 & 0.038 \\
\hline$(\mathrm{ng} / \mathrm{mL})$ & (1899.34-2425.22) & $\begin{array}{l}(2249.50- \\
3226.74)\end{array}$ & $\begin{array}{l}\text { (1375.07- } \\
2737.17)\end{array}$ & \\
\hline sCD163 & 892.98 & 1137.62 & 920.00 & 0.291 \\
\hline$(\mathrm{ng} / \mathrm{mL})$ & (765.64-1279.63) & $(847.93-1547.45)$ & (513.24-1262.92) & \\
\hline CCL2 & 318.85 & 225.68 & 176.64 & 0.074 \\
\hline$(p g / m L)$ & $(184.29-449.23)$ & $(143.88-413.65)$ & $(140.47-218.07)$ & \\
\hline
\end{tabular}

\section{Discussion}

Increased numbers of peripheral blood CD14+ monocytes/macrophages and activated CD14+/CD23+ monocytes/macrophages are seen during the acute stage of KD. They secrete high levels of TNFa and IL1 in acute KD. In our study, we documented increase in CD14+ monocytes, classical (CD14+CD16-) and intermediate (CD14++CD16+) monocytes during acute phase of KD that normalised in follow-up. Both early (CD69) and late (HLA-DR) activation markers in monocytes were also elevated in acute phase of KD that reduced in follow-up. There were significantly elevated levels of CCL2 in acute KD, as compared with post-IVIg and convalescent phase. We observed higher levels of CCL2 in patients with KD and febrile controls as compared to healthy controls.

In our cohort, we found significantly increased AMC in acute stages of KD as compared to healthy and febrile controls. Majority of the previous studies in KD have focussed on mononuclear cell counts rather than just monocyte counts. Matsubara et al. found no significant difference in mononuclear cell count between KD patients and control but monocyte count (CD14+) was significantly higher in patients with acute KD as compared to control subjects [10]. Several other studies have documented elevated levels of different serum cytokines (TNF-alpha, IL-1, and IL-6) that are reckoned to be produced by monocytes in the acute stage of KD [11] Matsuguma $C$ et al studied the difference in absolute monocyte counts between IVIg responsive and IVIg resistant groups. They found AMC to be significantly higher in IVIg responsive group as compared to IVIg resistant group [12]. 
Classical monocytes [CD14+ CD16-], which constitute the majority of monocytes, act as scavengers toward apoptotic cells and assist in resolving inflammation [13]. Our study showed a significant increase in absolute counts of classical monocytes [CD14+CD16-] in acute KD as compared to febrile and healthy controls. Intermediate monocytes [CD14++CD16+] express chemokine and TNF receptors and they are considered as pro-inflammatory form of monocytes [14]. Katayama et al. studied 28 patients with KD along with other febrile healthy controls. They found a significant increase in percentage as well as absolute numbers of CD14+CD16+ monocytes in acute KD as compared with convalescent KD and control subjects [15]. In our study, we have studied both intermediate monocytes [CD14++CD16+] and non-classical [CD14+CD16++] monocytes separately. On comparing acute KD with post-IVIg and followup samples, we also found a significant decrease in the absolute counts of intermediate monocytes [CD14++CD16+]. Non-classical monocytes [CD14+CD16++] are a minor subset of monocytes that have a proinflammatory role. These monocytes also have a role in surveillance of endothelial integrity and are thus referred to as patrolling monocytes [16]. In our study, we noted no difference in either the percentage or absolute counts of non-classical monocytes [CD14+CD16++] between acute KD, healthy and febrile controls. Burbano et al. found decreased percentage of non-classical monocytes [CD14+CD16++] in active SLE as compared to inactive SLE and healthy controls [17]. To the best of our knowledge, no study has studied nonclassical monocytes [CD14+CD16++] in $\mathrm{KD}$ and this area requires more research.

CD69 is an early activation marker that is expressed on all hematopoietic cells except RBCs. Crosslinking of this molecule leads to calcium influx and formation of both cyclooxygenase and lipoxygenase metabolites [18]. Many studies have been done on CD69 expression on T-lymphocytes in KD. However, studies on CD69 expression on monocytes in KD are lacking. CD69 expression on monocytes has been studied in various other diseases like Alzheimer's disease and sarcoidosis. In our cohort of KD patients, we found an increased percentage expression of CD69 in total monocytes (CD14+) in KD cases and febrile controls as compared to healthy controls suggesting the role of early monocyte activation in KD. HLA-DR is a late monocyte activation marker and is mainly seen on antigen presenting cells (APCs). HLADR is a surrogate marker for immune system stimulation and the expression of HLA-DR on monocytes has been shown to determine the efficacy of antigen presentation to T-helper cells [19]. Increased numbers of monocytes with high expression of HLA-DR have been shown in other inflammatory disorders like rheumatoid arthritis and inflammatory bowel disease [20-21].

On comparing acute KD with post-IVIg and convalescent stage we found a significantly higher percentage of total monocytes (CD14+) expressing the late activation marker (HLA-DR) in acute stage and post-IVIg as compared to convalescent stage. These findings indicate increased antigen presentation by monocytes in acute stage. One of the well-known mechanisms of IVIg is to decrease antigen presentation by downregulating MHC-II and dendritic cells and also to decrease T-lymphocyte expansion and to increase apoptosis of T-lymphocytes. Hence, decrease in HLA-DR expression could be related to immunomodulatory action of IVIg [22].

Several studies have documented changes in the levels of different activation markers of monocytes like sCD14, sCD163, CCL2/MCP-1 etc in patients with KD (Table 4). [5-8] In the present study, we 
documented significantly elevated levels of CCL2 in acute KD, as compared with post-IVIg and convalescent phase, and MCP-1/CCL2 levels were higher in patients with KD and febrile controls in comparison to healthy controls. These findings were consistent with the results of Asano et al. and Terai et al who observed significantly elevated circulating levels of MCP-1 in the acute phase of KD that drastically reduced after IVIg therapy. $[7,23]$ In our study, there was no significant rise in SCD14 and sCD163 levels in children with acute KD when compared with febrile and healthy controls. This is in contrast to the results obtained by Takeshita et al and Azuma et al who reported significant elevation in sCD14 levels and SCD163 levels respectively in children with acute KD compared with febrile and healthy controls. [5, 6] Apart from variation in ethnicity, the difference in duration of fever in children with KD enrolled in those studies can also possibly explain the contradictory results. While Takeshita et al and Azuma et al enrolled children with KD who had median fever duration of 7.5 days and 4 days respectively, we enrolled cases with KD with the median duration of fever of 9 days. It is possible that elevation of sCD14 and SCD163 levels occurs mostly in the first week of illness in KD that subsides in the second week.

Azuma et al also noted significant elevation in levels of SCD163 following IVIg therapy in initial IVIg responder group, however, the same observation was not noted in IVIg non-responder group. Authors speculated that IVIg could possibly detach CD163 from the surface of macrophages due to cross-linking of Fc-gamma receptors. [6] We also observed increase in SCD163 levels following IVIg therapy. However, the difference was not statistically significant. Small sample size of our cohort could have possibly resulted in not achieving the statistical significance.

Cheung et al. (2005) showed significant overexpression of CCL2/MCP1 genes in THP1 macrophages in children with CAAs who had past history of KD [24]. But this study was carried out in children with KD at mean 7 years after acute illness. These findings suggest that MCP-1 expression persisted to remain elevated in CAAs in KD.

Our study was unique as multiple monocyte activation markers were assayed in study population using methods like flow cytometry and ELISA. Another strength of this study was inclusion of different subsets of monocytes (classical, intermediate and non-classical) in analysis. Limitation of our study include a small sample size. However, this is understandable given that our study has to be carried out in a limited time span. Studying monocyte activation markers in a large multicentric cohort of patients would provide more convincing data on monocyte activation status in children with KD.

In conclusion, our study showed that both classical and intermediate monocytes along with early and late monocyte activation markers were elevated in acute phase of KD that subsided in follow-up. We also documented elevated levels of CCL2/MCP1 in acute stage of KD implying monocyte activation in the acute phase of KD. Our results suggest IVIg may decrease monocyte activation in KD, thereby controlling systemic inflammation. However, results of our study must bereplicated in larger cohorts with inclusion of 
more severe forms of KD to assess whether thedegree of monocyte activation could predict IVIg resistance or formation of CAAs.

\section{Materials And Methods Study Population}

We enrolled sixteen $(n=16)$ children diagnosed as KD in this prospective study from Pediatric Allergy Immunology Unit, Advanced Pediatrics Centre, PGIMER, Chandigarh, India (January 2019 to December 2020). All were diagnosed with KD according to the criteria enunciated by the American Heart Association (AHA), 2017. [9]

Sixteen $(n=16)$ age and sex matched healthy controls and sixteen $(n=16)$ febrile controls were enrolled from pediatric outpatient department and inpatient wards, respectively. Institutional Ethics Committee of Post Graduate Institute of Medical Education and Research, Chandigarh, approved the study (IEC/2019/001520 dt 09/08/2019 and IEC/2019/001448 dt 18/07/2019). Written informed consents were taken from the parents/guardians of all the participants. While ELISA for soluble markers of monocyte activation (SCD14, sCD163, CCL2) was done for all 16 patients, flow-cytometry assay for assessment of monocyte sub-populations and expression of monocyte activation markers (CD14, CD16, CD69, and HLA-DR) was performed in 11 out of 16 patients.

\section{Laboratory Blood Tests}

We estimated expression of CD16, CD69, and HLA-DR on CD14+ monocytes. Under sterile aseptic precautions, $2 \mathrm{ml}$ of peripheral venous blood was drawn by venepuncture into the Ethylene diamine tetraacetic acid (EDTA) vacutainer. Samples were collected at three different stages of illness in patients with KD- prior to administration of IVIg and aspirin, 24 hours after receiving IVIg, and 3 months after illness onset (convalescent stage). EDTA samples were processed immediately for flow cytometry assay. One hundred (100) $\mu \mathrm{l}$ of whole blood from the EDTA vacutainer was transferred in two falcon tubes. One tube was labeled as unstained (only blood) and other tube was immunolabelled with the relevant antibodies- CD45, CD14, CD16, CD69, and HLA-DR. The sample with labeled antibodies was then incubated in dark at room temperature for 30 minutes. After incubation, the erythrocytes were lysed twice using 1 x FACS lysing solution. The remaining WBCs were washed twice with the staining buffer. The expression of surface markers was then assessed by flow cytometry (Beckman Coulter, Navios, USA) using Kaluza software. Monocytes were gated on the forward/CD45 vs side scatter plot. Three different population of monocytes were selected as classical monocytes [CD14+CD16], intermediate monocytes [CD14++CD16+] and non-classical monocytes [CD14+CD16++]. (Figure 1) The percentage expression and median fluorescence intensity (MFI) of CD69 and HLA-DR were assessed on classical monocytes [CD14+CD16-], intermediate monocyte [CD14++CD16+] and non-classical monocytes [CD14+CD16 ++]. (Figure 1) 
We also measured plasma sCD14, sCD163, and CCL2 levels by ELISA. Plasma was extracted from the EDTA samples by centrifugation (3000 rpm for 5 minutes) and was stored at $-80^{\circ} \mathrm{C}$ refrigerator for future analysis. We used commercially available Quantikine ${ }^{\circledR}$ ELISA kits (R\&D Systems $®$ USA) in a fully automated ELISA machine (Infinite200 pro) and the results were read in the ELISA reader (Tecan).

\section{Statistical analysis}

Statistical analysis was performed using SPSS (Statistical Package for Social Sciences) software version 21.0 (SPSS Inc, Chicago). Quantitative variables were expressed as median and inter-quartile ranges (IQR) for skewed distribution. Kruskal-Wallis test was used for comparing variables amongst three independent groups. Mann-Whitney test was used for comparison between two independent groups. For comparing three repeated measures (acute KD, post-IVIG, and 3rd month follow-up samples), Friedman test was used. Wilcoxon-signed rank test was used for comparison between two repeated measures. A pvalue of less than 0.05 was considered significant.

\section{Declarations}

Sources of funding: ICMR (Indian Council of Medical Research) [No. 3/2/July-2019/PG-Thesis-HRD (31)] [No. 3/2/July-2019/PG-Thesis-HRD (32)]

Conflicts of interest: All authors declare there are no conflicts of interest

Statement: All methods were carried out in accordance with relevant guidelines and regulations.

Data availability/Availability of Data and Materials: The datasets used and/or analysed during the current study available from the corresponding author on reasonable request.

Informed consent: Written informed consent was obtained from parents of children included in this study for publication as case report

Consent for publication: All authors have given final approval for publication

Competing interests: None

\section{References}

1. Takahashi K, Oharaseki T, Yokouchi Y. Pathogenesis of Kawasaki disease: Pathogenesis of Kawasaki disease. Clinical \& Experimental Immunology. 2011 May;164:20-2.

2. Toubiana J, Poirault C, Corsia A, Bajolle F, Fourgeaud J, Angoulvant F, et al. Kawasaki-like multisystem inflammatory syndrome in children during the covid-19 pandemic in Paris, France: prospective observational study. BMJ. 2020;m2094. 
3. Manlhiot C, Mueller B, O'Shea S, Majeed H, Bernknopf B, Labelle M, et al. Environmental epidemiology of Kawasaki disease: Linking disease etiology, pathogenesis and global distribution. Kuo H-C, editor. PLOS ONE. 2018 Feb 7;13(2):e0191087.

4. Katayama K, Matsubara T, Fujiwara M, Koga M, Furukawa S. CD14 + CD16 + monocyte subpopulation in Kawasaki disease: CD14 + CD16 + monocytes in Kawasaki disease. Clinical \& Experimental Immunology. 2000;121:566-70.

5. Takeshita, Nakatani, Tsujimoto, Kawamura, Kawase, Sekine. Increased levels of circulating soluble CD14 in Kawasaki disease: Increased soluble CD14 in KD. Clinical \& Experimental Immunology. 2000;119:376-81.

6. Azuma Y, Suzuki Y, Okada S, Matsuguma C, Wakiguchi H, Ohnishi Y, et al. Utility of Soluble CD163 in the Clinical Management of Patients With Kawasaki Disease. Front Pediatr. 2020;8:148.

7. Terai M, Jibiki T, Harada A, Terashima Y, Yasukawa K, Tateno S, et al. Dramatic decrease of circulating levels of monocyte chemoattractant protein-1 in Kawasaki disease after gamma globulin treatment. J Leukoc Biol. 1999;65:566-72.

8. Shikishima Y, Saeki T, Matsuura N. Chemokines in Kawasaki disease: measurement of CCL2, CCL22 and CXCL10. Asian Pac J Allergy Immunol. 2003;21:139-43.

9. McCrindle BW, Rowley AH, Newburger JW, Burns JC, Bolger AF, Gewitz M, et al. Diagnosis, Treatment, and Long-Term Management of Kawasaki Disease: A Scientific Statement for Health Professionals From the American Heart Association. Circulation. 2017 Apr 25;135(17).

10. Matsubara T, Ichiyama T, Furukawa S. Immunological profile of peripheral blood lymphocytes and monocytes/macrophages in Kawasaki disease. Clinical and Experimental Immunology. 2005 Sep;141(3):381-7.

11. Maury CP, Salo E, Pelkonen P. Circulating interleukin-1 beta in patients with Kawasaki disease. The New England journal of medicine. 1988;319(25):1670-1.

12. Matsuguma C, Wakiguchi H, Suzuki Y, Okada S, Furuta T, Ohnishi Y, et al. Dynamics of immunocyte activation during intravenous immunoglobulin treatment in Kawasaki disease. Scandinavian Journal of Rheumatology. 2019 Nov 2;48(6):491-6.

13. Koizumi K, Hoshiai M, Katsumata N, Toda T, Kise H, Hasebe $Y$, et al. Infliximab regulates monocytes and regulatory T cells in Kawasaki disease. 2018;7.

14. Hijdra D, Vorselaars ADM, Grutters JC, Claessen AME, Rijkers GT. Phenotypic Characterization of Human Intermediate Monocytes. Front Immunol. 2013;4.

15. Katayama K, Matsubara T, Fujiwara M, Koga M, Furukawa S. CD14+CD16+ monocyte subpopulation in Kawasaki disease. Clinical and Experimental Immunology. 2000 Sep;121(3):566-70.

16. Ginhoux F, Jung S. Monocytes and macrophages: developmental pathways and tissue homeostasis. Nat Rev Immunol. 2014 Jun;14(6):392-404.

17. Burbano C, Vasquez G, Rojas M. Modulatory Effects of CD14+CD16++ Monocytes on CD14++CD16Monocytes: A Possible Explanation of Monocyte Alterations in Systemic Lupus Erythematosus: 
Effects of CD14+CD16++ Monocytes on CD14++CD16- Monocytes. Arthritis \& Rheumatology. 2014 Dec;66(12):3371-81.

18. Testi R. Triggering of Human Monocyte Activation through CD69, a Member of the Natural Killer Cell Gene Complex Family of Signal Transducing Receptors. 1994;6.

19. Carero A, Hoet P, Nemery B, Schoeters G. Increased HLA-DR expression after exposure of human monocytic cells to air particulates. Clinical and experimental allergy: journal of the British Society for Allergy and Clinical Immunology. 2002 Mar 1;32:296-300.

20. Ridley MG, Kingsley G, Pitzalis C, Panayi GS. Monocyte activation in Rheumatoid arthritis: evidence for in situ activation and Differentiation in joints. Rheumatology. 1990;29(2):84-8.

21. Tillinger W, Jilch R, Waldhoer T, Reinisch W, Junger W. Monocyte Human Leukocyte Antigen-DR Expression-A Tool to Distinguish Intestinal Bacterial Infections From Inflammatory Bowel Disease?: Shock. 2013 Aug;40(2):89-94.

22. Negi V-S, Elluru S, Sibéril S, Graff-Dubois S, Mouthon L, Kazatchkine MD, et al. Intravenous Immunoglobulin: An Update on the Clinical Use and Mechanisms of Action. J Clin Immunol. 2007 May;27(3):233-45.

23. Asano, Ogawa. Expression of Monocyte Chemoattractant Protein-1 in Kawasaki Disease: The AntiInflammatory Effect of Gamma Globulin Therapy. Scand J Immunol. 2000;51:98-103.

24. Cheung Y-F, Karmin O, Tam SCF, Siow YL. Induction of MCP1, CCR2, and iNOS Expression in THP-1 Macrophages by Serum of Children Late After Kawasaki Disease. Pediatr Res. 2005;58:1306-10.

\section{Table}

Table 4 is available in the Supplemental Files section.

\section{Figures}



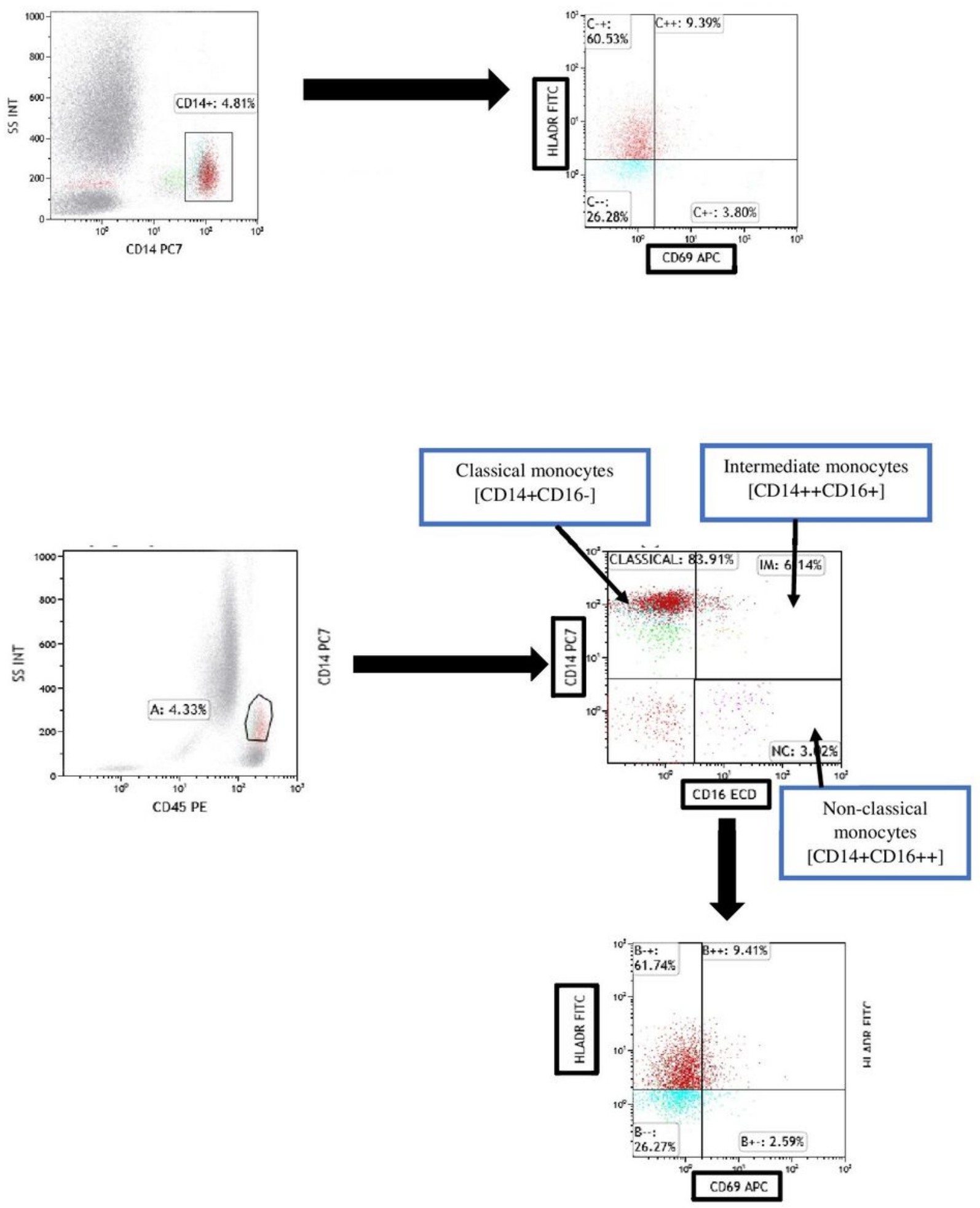

Figure 1

Gating Strategy of our study 

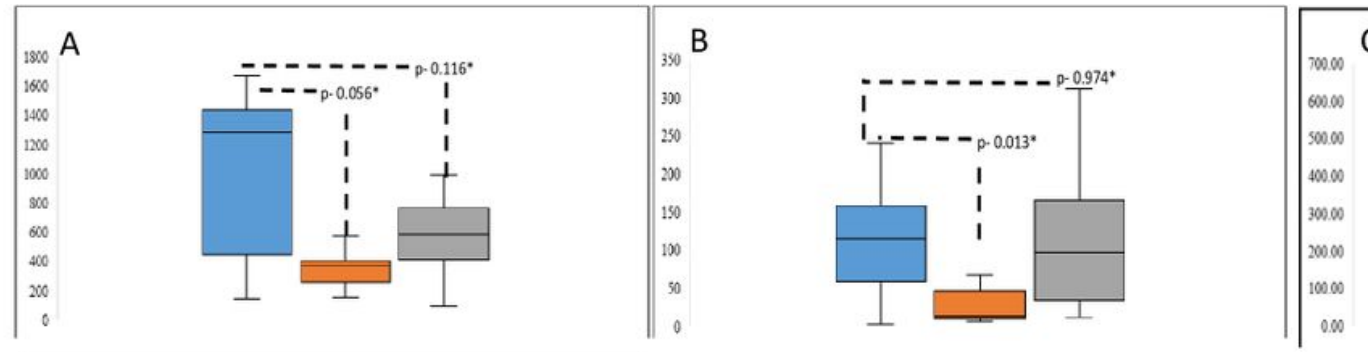

C
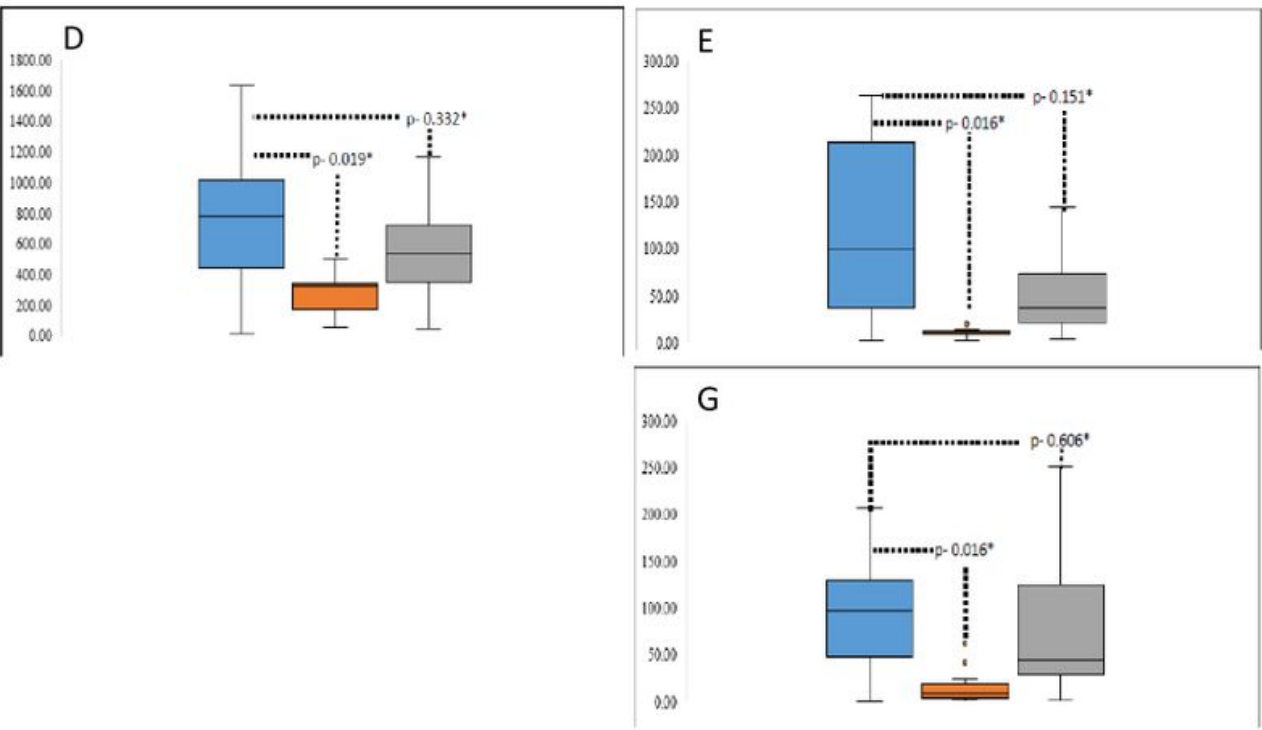

$\mathrm{F}$

5000
4500
4500
3500
3000
2500
2000
1500
1000
500

200

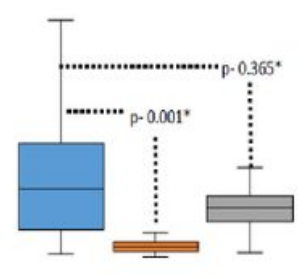

$\square$ Acute KD $\square$ Healthy control $\square$ Febrile control

Figure 2

Comparison of monocyte subpopulations and monocyte activation markers studied in acute KD, febrile controls, and healthy controls

(A) Comparison of classical monocytes [CD14+CD16] (absolute counts) (B) Comparison of intermediate monocytes [CD14++CD16+] (absolute counts) (C) Comparison of absolute number of CD14+ monocytes that showed CD69 expression (D) Comparison of absolute number of CD14+ monocytes that showed HLA-DR expression (E) Comparison of absolute counts of classical monocytes [CD14+CD16-] showing CD69 expression (F) Comparison of absolute counts of intermediate monocytes [CD14++CD16+] showing CD69 expression (G) Comparison of absolute counts of intermediate monocytes [CD14++CD16+] showing HLA-DR expression 

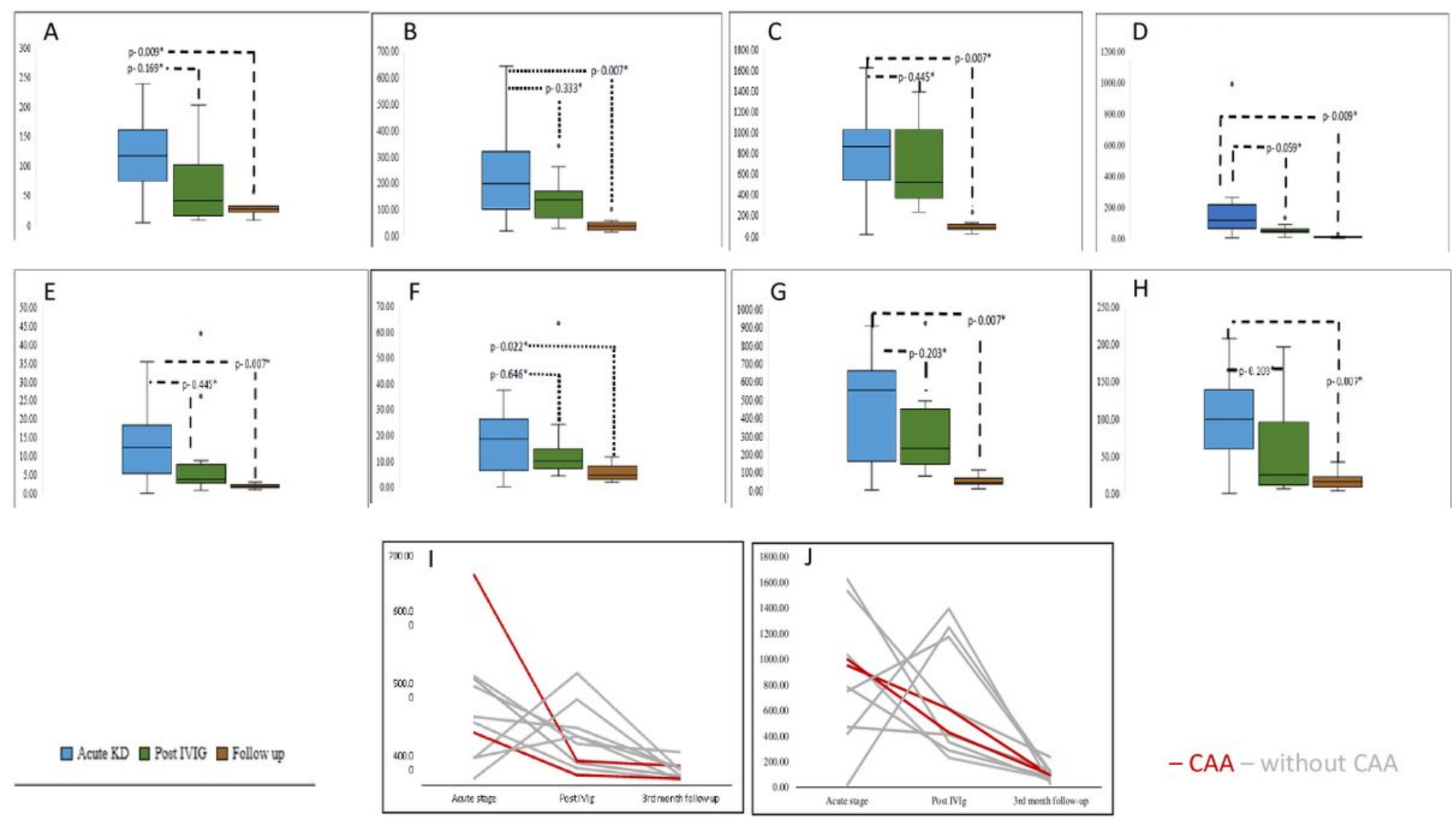

Figure 3

Comparison of monocyte subpopulations and monocyte activation markers studied in acute KD, PostIVIg, and $3^{\text {rd }}$ month follow-up

(A) Comparison of absolute counts of Intermediate monocytes [CD14++CD16+] (B) Comparison of absolute counts of CD14+ monocytes showing CD69 expression (C) Comparison of absolute counts of CD14+ monocytes showing HLA-DR expression (D) Comparison of absolute counts of Classical monocytes [CD14+CD16-] showing CD69 expression (E) Comparison of absolute counts of Intermediate monocytes [CD14++CD16+] showing CD69 positivity (F) Comparison of absolute counts of Non-Classical monocytes [CD14+CD16++] showing CD69 expression (G) Comparison of absolute counts of Classical monocytes [CD14+CD16-] showing HLA-DR expression $(H)$ Comparison of absolute counts of Intermediate monocytes [CD14++CD16+] showing HLA-DR expression (I) Trend of Absolute counts of CD14+ monocytes expressing CD69 in KD patients with CAA as compared to patients without CAAs (J) Trend of Absolute counts of CD14+ monocytes expressing HLA-DR in KD patients with CAA as compared to patients without CAAs

\section{Supplementary Files}

This is a list of supplementary files associated with this preprint. Click to download. 
- Table4.docx

- Supplementaryfile.docx 